\title{
OVARIECTOMISED AND LONG TERM INDUCED (OLI) RAT MODEL FOR PRECLINICAL ASSESSMENT OF OSTEOPOROTIC THERAPEUTICS
}

\author{
Chandran Sunitha ${ }^{1}$, V.S. Harikrishnan ${ }^{2}$ and John Annie ${ }^{1 *}$ \\ ${ }^{1}$ Transmission Electron Microscopy Laboratory, Biomedical Technology Wing, Sree Chitra Tirunal Institute for Medical Sciences \& Technology, \\ Thiruvananthapuram, India- 695012 \\ ${ }^{2}$ Division of Laboratory Animal Science, Biomedical Technology Wing, Sree Chitra Tirunal Institute for Medical Sciences \& Technology, \\ Thiruvananthapuram, India- 695012
}

\section{ABSTRACT}

Osteoporosis affects millions of people, especially the aged population and numerous researchers focus on developing novel pharmacotherapeutic agents for the prevention and treatment of osteoporosis. Animal models have become an indispensable part of the preclinical assessment of osteoporotic therapeutics and rat models have been widely preferred. Majority of studies so far reported have used young or short-term induced rat models, but they do not mimic the pathological condition in senile osteoporosis. Hence this study has focused on developing an ovariectomised and long term induced (OLI) aged rat model for the preclinical assessment studies. Long term osteoporosis was induced in Wistar rats by ovariectomy followed by the maintenance period of 10 months. Histological evaluation and Micro-CT analysis have substantiated the extensive bone loss in the OLI rats compared to Control, ovariectomised and short term induced (OSI) rat model. OLI rats also exhibited impairment in the healing of $3 \times 1.5 \mathrm{~mm}$ femoral cortical bone defect even after 8 weeks of surgery. Histomorphometric analysis indicated that OLI rats exhibited a poor regeneration efficiency (RE) ratio of $0.67 \pm 0.2$ compared to $0.95 \pm 0.1$ in the Control group. Chronic bone loss in the OLI rats together with the impaired osteogenic efficacy has been successfully demonstrated which proposes OLI rat models to be utilized for investigating novel pharmacotherapies or novel biomaterials intended for osteoporotic applications.

Keywords: Osteoporosis, Rat osteoporotic model, long-term osteoporosis induction, estrogen deficiency.

\section{Article Info}

Received 12 Feb 2017; Review Completed 23 Feb 2017; Accepted 24 Feb 2017, Available online 15 March 2017

\section{Cite this article as:}

Sunitha C, Harikrishnan VS, Annie J, Ovariectomised and long term induced (OLI) rat model for preclinical assessment of osteoporotic therapeutics, Journal of Drug Delivery and Therapeutics. 2017; 7(2):36-43. DOI: http://dx.doi.org/10.22270/jddt.v7i2.1400

*Address for Correspondence

Dr. Annie John, Transmission Electron Microscopy Laboratory, Biomedical Technology Wing, Sree Chitra Tirunal Institute for Medical Sciences \& Technology, Thiruvananthapuram, India- 695012

Tel: 91 9495340030, Fax: 91-471-2341814, E-mail: karippacheril@gmail.com

\section{INTRODUCTION}

Osteoporosis has become a common skeletal disease and usually makes its appearance during the late age ${ }^{1}$. At 50 years of age, 4 in 10 women succumb to osteoporosis related fractures and a prior fracture increases further fracture incidence by 10 fold, thus making osteoporotic treatment among the aged population an orthopaedic challenge ${ }^{2}$. The alleviating number in the elderly population with associated osteoporotic fracture incidence has raised much socio economic concerns too ${ }^{3}$. New treatment strategies are therefore required to reduce and prevent the onset of disease and thereby incidence of fractures. 
An appropriate animal model is indispensable for evaluating the safety and efficacy of any new modality prior to its clinical application. Rat osteoporotic model has been considered the optimal model for osteoporosis research, as it resembles human pathological condition in skeletal responses to depletion of estrogen and pharmacological effects on bone turnover $^{4}$. There are various methods of inducing osteoporosis in rats such as ovariectomy, low calcium diet and immobilization 5. Aging predisposes the skeleton to excessive bone loss in conditions of senile osteoporosis. Senile osteoporosis is associated with progressive changes in bone remodelling, mainly by decreasing bone formation relative to bone resorption ${ }^{6}$. However literature review suggests that most animal model-based osteoporosis research has been carried out in osteoporotic models induced by ovariectomy in young rats or by short term induction period of 3-6 months. Significant age-related changes were not observed in bone mineral content (BMC) and bone mineral density (BMD) in Sham and ovariectomised rats posts 3-6 months of induction ${ }^{7}$. Kubo et al., has also reported the weak effect of short term induced osteoporosis (30 weeks post ovariectomy) in the mechanical properties and in early phases of bone defect healing ${ }^{8}$. A more appropriate osteoporotic model would be to have long term osteoporosis induced animals, so as to better mimic the chronic bone loss of the aged human clinical pathology. Hence our objective was to develop a novel model with chronic bone loss induced by ovariectomy followed by a prolonged induction period. It was also of interest to evaluate the effect of long term induction on the bone healing efficacy in the OLI model.

\section{MATERIALS AND METHODS:}

\section{Ethical statement:}

All in vivo studies were performed as per the guidelines and recommendations of the Committee for the Purpose of Control and Supervision of Experiments on Animals (CPCSEA), India and with the approval of the Institute Animal Ethics Committee (IAEC, India).

Animals were housed in individually ventilated cages at $22 \pm 2^{\circ} \mathrm{C}$ and $55 \pm 10 \%$ relative humidity ( $\left.\mathrm{RH}\right)$. Light levels measured (at 1 meter high) less than 300 lx, and a 12:12 hour dark/light pattern was maintained. Animals were fed with standard pelleted rat feed and drinking water ad libitum. The health of the animal colony was monitored as per Federation for Laboratory Animal Science Associations (FELASA) guidelines for parasitology 9 , a written protocol developed from international recommendations for bacteriology, protozoology and virology and was stamped negative for infectious agents. The study also followed the Animal Research Reporting of Experiments (ARRIVE) guidelines for the execution, evaluation and reporting of the in vivo experiments ${ }^{10}$.
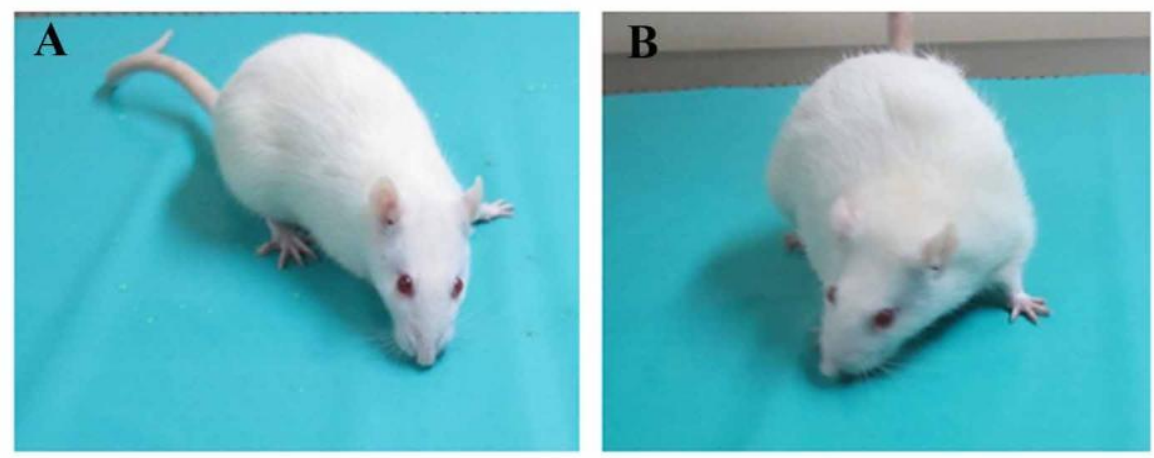

Figure 1: Rat model: (A) and (B) Control rat and ovariectomised and long term induced (OLI) model.

\subsection{Bilateral ovariectomy:}

Osteoporosis was induced in female Wistar rats - Sctb: WI rats $(\mathrm{n}=9)$ (outbred Wistar rats) aged 3 months, weighing approximately $200 \mathrm{~g}$, by bilateral ovariectomy. Animals were further grouped as: ovariectomised and short term induced (four months, $n=3$ ) (OSI), and ovariectomised and long term induced (ten months, $\mathrm{n}=6$ ) (OLI). Animals aged 13 months were included within the Control group ( $\mathrm{n}=6$ ) (figure 1A). Surgeries were performed under general anesthesia using $80 \mathrm{mg} / \mathrm{kg}$ ketamine (Anket, Neon Lab, India) and $5 \mathrm{mg} / \mathrm{kg}$ xylazine (Xylaxin, Neon Lab, India). Briefly, the surgical site was clipped and prepared with 5\% Povidone iodine solution (Betadine, Win Media care, India). The skin and abdominal musculature of the abdominal wall were incised at the flank level. Using a pair of fine tweezers, the peritoneal fat pad was grasped and exteriorized to view the ovary and uterine horn on each side. The vascular supply to the ovary was clamped using mosquito forceps and the ovary was excised. After clamping the distal region of the uterine horn, a portion of the uterine horn was also excised. The clamps were removed and haemostasis was confirmed. The tissue and fat pad were replaced within the peritoneal cavity using the blunt end of vascular forceps and the skin was closed using 3-0 braided silk (Mersilk, Johnson \& Johnson, USA) in a simple interrupted pattern. Povidone iodine solution was applied daily for 7 postoperative days until the sutures were removed. Post operatively animals received subcutaneous injections of 
Analgesic-Meloxicam (Melonex, Indian Immunologicals Ltd, India) at $1 \mathrm{mg} / \mathrm{kg} \mathrm{S} / \mathrm{C}$ once daily and Buprenorphine (Buprigesic, Neon Lab, India) at $0.05 \mathrm{mg} / \mathrm{Kg} \mathrm{i} / \mathrm{m}$ twice daily for 7 days.

Good nursing support was provided along with the medications, by providing a soft and absorbent bedding to prevent postsurgical infection. Rats were also provided with pre-moistened feed pellets for the first three days after surgery and then high-quality feed (such as sprouted Bengal gram) was provided as soon as the animals recovered, to prevent weight loss and to support their high nutritional demands. Ovariectomised rats were further maintained for 4 months and 10 months to develop OSI and OLI groups respectively (figure 1B).

\subsection{Osteoporotic model assessment:}

\subsubsection{Serum Calcium level analysis:}

Serum was isolated from Control, OSI and OLI rats $(\mathrm{n}=3$ each) at 0,4 and 10 months following ovariectomy. Serum Calcium concentrations were measured using the kit Arsenazo III method (Cat No: BLT0001 Erba, Germany). Calcium concentration was then compared between the induced and age matched Control groups.

\subsubsection{Micro CT evaluation:}

Animals ( $\mathrm{n}=3$ each from OSI and OLI group) were euthanized at 4 and 10 months respectively, following osteoporosis induction and proximal tibiae were extracted. Muscle tissue was gently scraped off and bones were fixed in $10 \%$ neutral buffered formalin. Metaphyseal cancellous bone at the proximal tibiae from Control, OSI and OLI groups were scanned using micro CT desktop scanner CT 40 (Scanco Medical AG, Switzerland) set at $70 \mathrm{kVp}$ and $114 \mu \mathrm{A}$. A region of interest (ROI) was chosen by selective contouring of the trabecular bone image slices and three dimensional volumetric model reconstructions were performed using in built software (version - 6.5). Bone parameters such as trabecular number (Tb.N.), trabecular spacing (Tb.Sp.) and bone density were assessed and further evaluated.

\subsubsection{Histological evaluation:}

For histological analysis formalin fixed bone samples $(n=3)$ were decalcified using $12 \%$ Ethylene di-amine tetra acetic acid (EDTA) and underwent a series of dehydration processes in alcohol prior to paraffin embedding. Sections from the paraffin embedded blocks were cut using a rotary microtome (Leica RM 2255, Germany), stained using Hematoxylin and Eosin (H\&E) and evaluated by light microscopy (Leica DM 6000, Germany).

2.3. Evaluation of effect of long term osteoporosis induction in the OLI model:

\subsubsection{Surgical procedure:}

To assess the effect of long term osteoporosis induction on bone healing, femoral bone defects of $3 \mathrm{~mm} \times 1.5 \mathrm{~mm}$ were made in OLI rats $(\mathrm{n}=3$ rats) and compared with that of the Control animals. Under Xylazine-Ketamine anaesthesia $(5 \mathrm{mg} / \mathrm{Kg}$ and $80 \mathrm{mg} / \mathrm{kg}$, respectively) distal femur was exposed through a lateral approach and a bone defect was created with a surgical drill (Marathon, Saeyang microtech, Korea). The muscle flap was then replaced and incision was sutured.

Postoperatively, all animals received antibiotic injections intramuscularly for 7 days. Animals also received subcutaneous injections of Meloxicam (Melonex, Indian Immunologicals Ltd, India) at $1 \mathrm{mg} / \mathrm{kg} \mathrm{S} / \mathrm{C}$ once daily and Buprenorphine (Buprigesic, Neon Lab, India) at $0.05 \mathrm{mg} / \mathrm{Kg} \mathrm{I} / \mathrm{M}$ twice daily.

\subsubsection{Histological evaluation of bone defect healing:}

Animals were euthanized 8 weeks post surgery and the defect sites, along with the adjacent bone, were extracted without disturbing the defect area. Retrieved bone samples were then formalinfixed and underwent a series of dehydration steps in alcohol, followed by infiltration and embedding in methyl methacrylate (MMA). Plastic embedded blocks were then sectioned using a high-speed precision saw (Isomet TM 2000 Precision Saw, Buehler, USA). Plastic sections were then manually polished down to 70-90 microns using a variable speed grinder/polisher (Ecomet 3000, Buehler, USA) and stained with Stevenal's blue and van Gieson's picrofuchsin ${ }^{11}$. Histological sections were then micrographed using the light microscope (Leica DM 6000, Germany).

Histomorphometry was performed to quantify bone defect healing in the Control and OLI animals. Consecutive histological sections $(n=3-4$ plastic sections) from randomly selected blocks were blindly evaluated using histomorphometry image analyzing software (Leica Qwin, Germany). Regeneration ratio (RE) (ratio of New Bone formed per Total area: [NB/TA]) was calculated from each section to assess the healing efficacy.

\subsection{Statistical analysis:}

All data included in the study were expressed as mean \pm standard deviation (SD). Results of the biochemical analyses of serum Calcium concentration and estimation of trabecular bone loss based on micro CT scans were evaluated using 2 way ANOVA (with multiple comparisons) in Graph pad prism (version 6.01). Statistical comparisons of histomorphometric data were performed using unpaired t tests. A p-value $<0.05$ was considered statistically significant in all analysis. 


\section{RESULTS:}

\subsection{Osteoporotic model evaluation:}

All animals (Control, OSI and OLI) recovered from surgery without any adverse effect or inflammatory responses.

\subsubsection{Evaluation of serum Calcium concentration:}

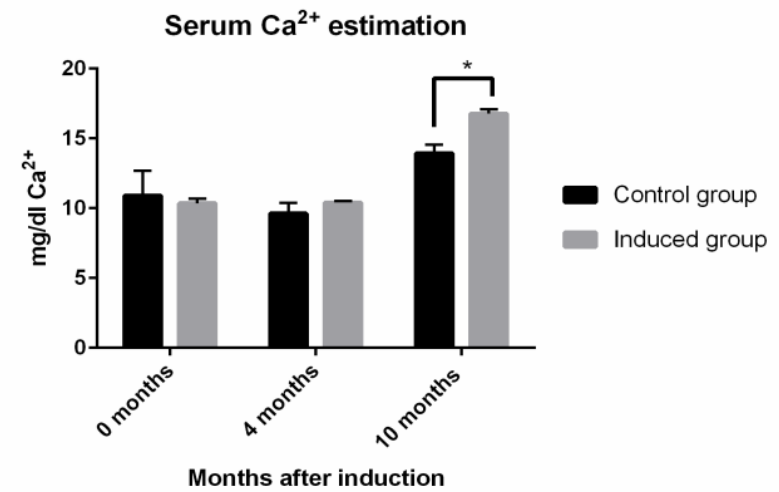

of the osteoporosis induced rat model post 0,4 and 10 months of induction compared to age matched Control group. (*- significant, actual $\mathrm{p}$ value0.0218).

Biochemical analyses (figure 2) indicated that serum Calcium concentration in ovariectomised rats was not significantly different from that of the age matched Control group at 0 and 4 months, indicating not much bone loss with short term induction period. Control group rats exhibited a Calcium concentration of $10.91 \pm 1.76 \mathrm{mg} / \mathrm{dl}$ and $9.632 \pm 0.76 \mathrm{mg} / \mathrm{dl}$, whereas the induced group animals exhibited a Calcium concentration of $10.35 \pm 0.3 \mathrm{mg} / \mathrm{dl}$ and $10.41 \pm 0.74 \mathrm{mg} / \mathrm{dl}$ at 0 and 4 months respectively. The induced rats exhibited a Calcium concentration of $16.77 \pm .30 \mathrm{mg} / \mathrm{dl}$ post 10 months of induction, which was significantly higher than that of aged matched Control rats, which exhibited a Calcium concentration of 13.94 \pm .90 $\mathrm{mg} / \mathrm{dl}$ ( $\mathrm{p}$ value -0.0218 ), indicating extensive bone loss.

Figure 2: Biochemical analysis: Graphical representation of Calcium concentration in the serum

\subsubsection{Evaluation of bone loss using micro CT:}
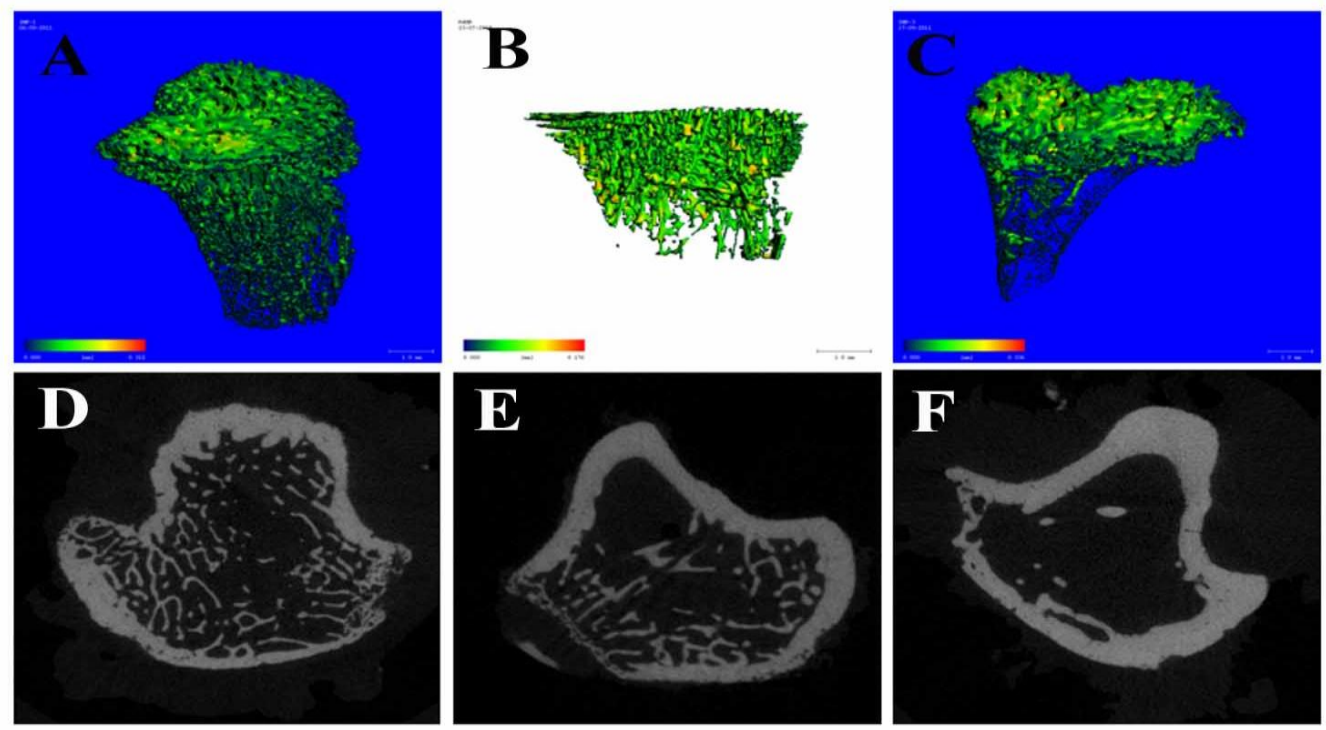

Figure 3: Osteoporotic model evaluation using micro CT scans: (A)-(C) depict the 3D trabecular thickness profile of the proximal tibial metaphysis from Control, OSI and OLI groups. Thinner trabeculae with less trabecular bone volume were evident in OSI and OLI groups. But extensive trabecular disruption was evident in the OLI group. (Color coding represents trabecular thickness thickness increases from green to red).(D), (E) and (F) depict random 2D slices from Control, OSI and OLI groups indicating the extent of trabecular bone disruption in OLI rats, when compared to Control and OSI rats.

In Control and OSI rats, trabecular bone (represented in greenish yellow color) was seen more prominent and extended downwards from the tibial plateau (figure $3 \mathrm{~A}$ and $3 \mathrm{~B}$ respectively). In the OLI rats, trabecular bone was evident only at the proximal tibial plateau, indicative of extensive trabecular disruption and bone loss. Random 2D slices generated from micro CT scans also depicted the extent of trabecular disruption among the different groups. In the OSI rat model (figure 3E) trabecular network was evident with slight disruption compared to a mesh-like network in the Control group (figure 3D), whereas in the OLI rat model (figure $3 \mathrm{~F}$ ) a few sprouts of trabecular network was only evident indicative of excessive bone loss compared to other groups. 
(A) Evaluation of trabecular number (Tb.N.)

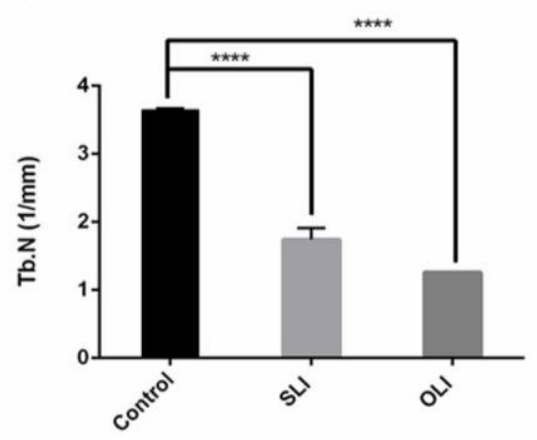

(B) Evaluation of trabecular spacing (Tb.Sp.)

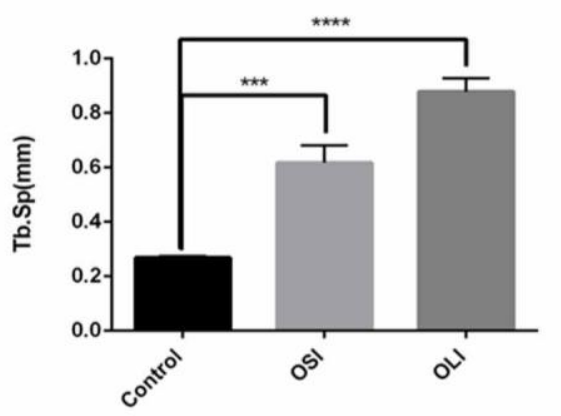

(C) Evaluation of Mean density of bone

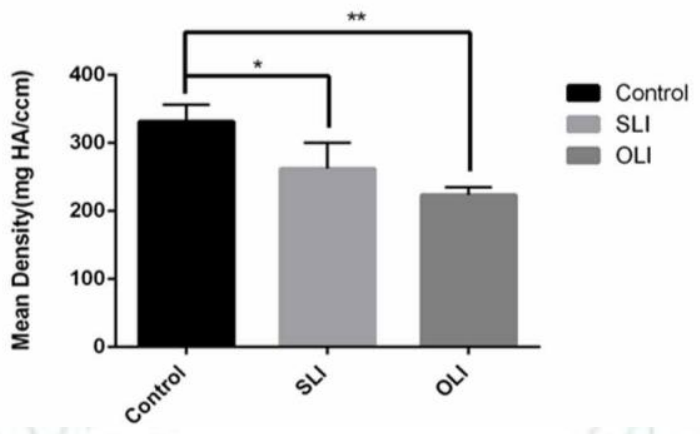

Figure 4: Quantitative evaluation of bone loss using micro CT scans: Decrease in trabecular number (Tb.N.) in the proximal tibial region from Control, OSI and OLI groups are represented in (A). Increase in trabecular spacing (Tb.Sp.) in the proximal tibial region of OLI group, compared to Control and OSI groups is depicted in (B). Figure $4 \mathrm{C}$ indicate the decline in bone density $\left(\mathrm{mg} \mathrm{HA} / \mathrm{cm}^{3}\right)$ in the OLI and OSI groups, but more significant change was evident in the OLI group, thereby confirming the significance of long term induction period in rats. (Fig A - Control vs. OSI $* * * * \mathrm{p}$ value $<0.0001$, Control vs. OLI $* * * * \mathrm{p}$ value $<0.0001$; fig $\mathrm{B}-\mathrm{Control}$ vs. OSI $* * * \mathrm{p}$ value 0.0002 , Control vs. OLI $* * * * \mathrm{p}$ value $<0.0001$; fig $\mathrm{C}-$ Control vs. OSI $* \mathrm{p}$ value 0.0362 , Control vs. OLI $* * \mathrm{p}$ value $<0.0051)$

Micro CT evaluation (figure 4A) indicated OSI and OLI rats to have a trabecular number (Tb.N.) of $1.73 \pm .17 / \mathrm{mm}(* * * * \mathrm{p}$ value $<.0001)$ and $1.26 \pm .04 / \mathrm{mm}$ $(* * * *$ v value $<.0001)$ respectively, compared to Control rats with Tb.N. of $3.65 \pm .01 / \mathrm{mm}$. Significant decline in Tb.N. was noted in the osteoporosis induced groups. Trabecular Spacing (Tb.Sp.) (figure 4B) was found to be $0.88 \pm .03 \mathrm{~mm}(* * * * \mathrm{p}$ value $<.0001)$ for OLI rats and $0.62 \pm .02 \mathrm{~mm}(* * * \mathrm{p}$ value 0.0002$)$ for OSI rats, compared to Control rats with Tb.Sp value of $0.27 \pm .003 \mathrm{~mm}$. Trabecular spacing seemed to increase with advancing induction period and significant high value in the OLI rats indicate that trabecular bone in the tibial head has been almost replaced with void spaces indicating trabecular disruption. Evaluation of trabecular bone density (figure 4C) also indicated a highly significant bone loss in the OLI animals, compared to other groups. OLI rats exhibited a significantly low mean density of $223.2 \pm 6.5 \mathrm{mg} \mathrm{HA} / \mathrm{cm}^{3}$ (**p value 0.0051 ) whereas the OSI rats and Control rats exhibited a value of $261.82 \pm 38.21 \mathrm{mg} \mathrm{HA} / \mathrm{cm}^{3}$ (*p value 0.0362) and $331.1 \pm 14.54 \mathrm{mg} \mathrm{HA} / \mathrm{cm}^{3}$. Trabecular bone parameters indicated chronic bone loss in the OLI animals, compared to OSI and Control rats.

\subsubsection{Histological evaluation of trabecular bone loss:}
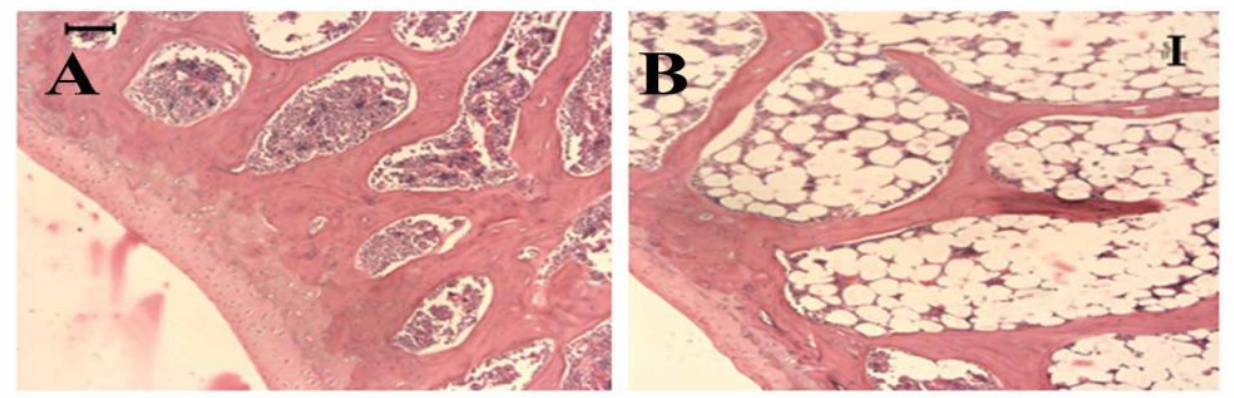

Figure 5: Histological evaluation of bone loss. H\&E-stained proximal tibial sections of OSI (A) and OLI rats (B) depict trabecular bone disruption. More void spaces with less trabeculae were evident in the OLI group. (Scale bar equivalent to $100 \mu \mathrm{m}$ ). 
Histological evaluation of H\&E stained sections of the tibial bone depicted a prominent trabecular network in the OSI group animals (figure 5A), whereas, in the OLI animals the trabecular network was noticeably thinner and surrounded by large void areas, indicating the extent of bone loss (figure 5B).

\subsection{Histological evaluation of bone defect healing:}
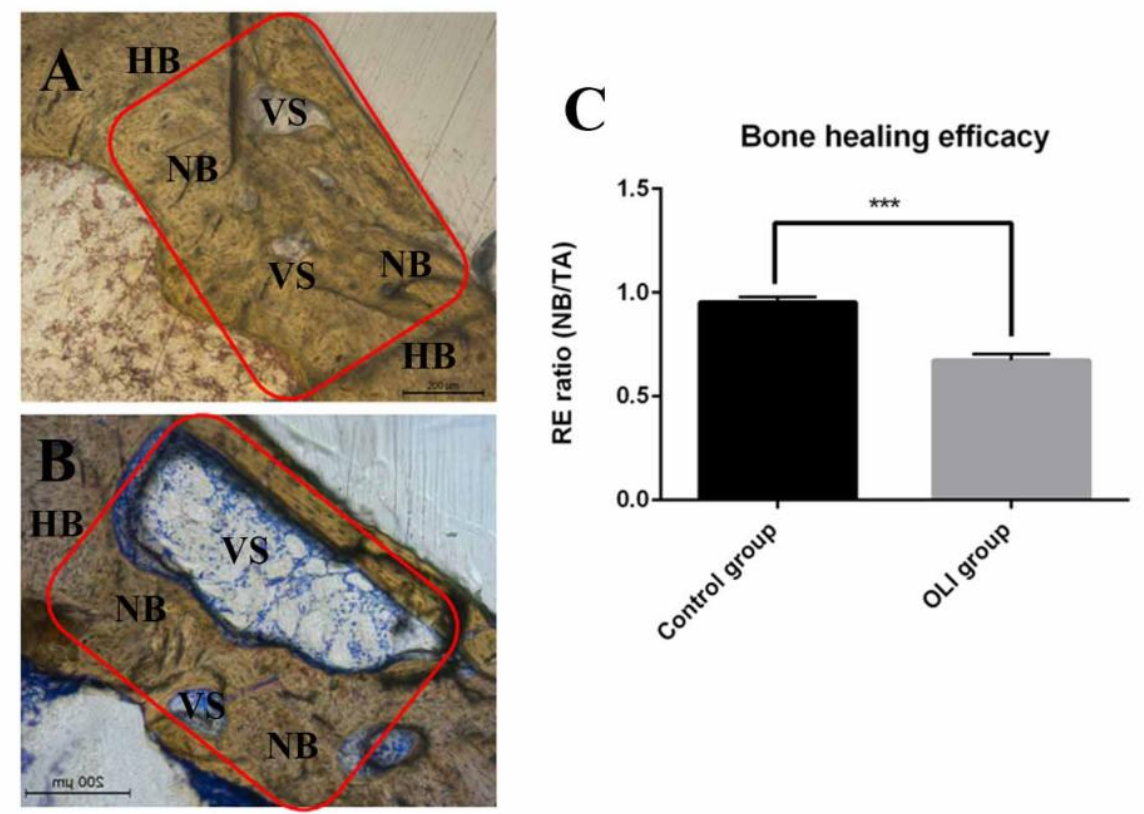

Figure 6: Histological evaluation of bone defect healing: (A) \& (B) shows Stevenal's blue and van Gieson's picrofuchsin stained plastic sections depicting in vivo healing in Control and OLI rats, 8 weeks after surgery. Large void spaces in the defect area (defect area - framed in red box) in the OLI rats (B) indicates delayed bone healing. In Control rats (A) the defect area was almost bridged by de novo osteoid matrix. Histomorphometry analysis (C) in terms of regeneration efficiency ratio indicated impaired healing in OLI animals, compared to Control group. (HB - Host Bone, NB - New Bone, VS - Void Space, TA - Total Area, RE Regeneration Efficiency $=$ New Bone/Total Area). ((A) \& (B) scale bar equivalent to $200 \mu \mathrm{m}$. (C) - *** significant, p value 0.0003 )

Histological evaluation carried out at eight weeks indicated reduced bone regeneration efficiency in OLI rats. In Control group (figure 6A), the bone defect area was almost bridged and filled with de novo osseous tissue. But in the OLI group, (figure 6B) even after eight weeks of surgery cortical bridging and closure of the defect area was not evident. Loosely arranged osteocytes (immature bone organization) could be distinguished in the de novo bone matrix in the control animals. But more void spaces with scarce new bone formation were evident in the OLI rats, indicative of poor healing. Further, histomorphometric analyses (fig 6C) indicated that OLI rats had a significantly lower RE ratio $(0.67 \pm .02)$ compared to age-matched Control animals ( $\mathrm{RE}$ ratio of $0.95 \pm .0 ; \mathrm{p}=0.0003)$, further supporting the effect of long term induction period in bone healing.

\section{DISCUSSION:}

The effect of long-term estrogen deficiency in rat models, to better mimic the clinical situation of senile osteoporosis in postmenopausal women, has not been explored. Most in vivo studies employ a short term period (3-4 months) of estrogen deficiency in rats for osteoporotic induction ${ }^{12,13}$ and different outcomes have been reported. Aging affects bone quality and has a major influence on skeletal morbidity and mobility ${ }^{14}$. Literature suggest that young rat skeletons are not to be considered as an appropriate model for senile osteoporosis, as bone turnover in these animals is primarily modelling (bone formation and bone resorption at specific sites), whereas in human osteoporosis, bone turnover is considered to be remodelling (formation and resorption occur in a cyclic manner at the same site) ${ }^{15}$. However, rat skeletal homeostasis is predicted to exhibit a gradual transition from modelling to remodelling with aging suggesting aged rat models to better mimic clinical pathology 16 . Therefore research on therapeutic modalities should consider the bone site and age of the rat model to be used. Rats at 6 months old inhibit rapid and linear bone growth as a sign of attaining maturity and maintaining Calcium haemostasis ${ }^{17}$. Fukuda and Iida (2004) have predicted 12 months old Wistar rats to exhibit age related bone loss in the proximal tibial metaphysis ${ }^{18}$. Accordingly, the significantly increased serum Calcium level in the 10 months osteoporosis induced OLI animals compared to SLI animals suggest the significance of long term induction period in view of 
clinical senile pathology. An increase in serum calcium concentration in normal rats at 13 months age has been reported elsewhere, indicating the effect of aging in bone loss ${ }^{19,20}$. The OLI models used in the study may be considered to have the etiological factors associated with estrogen deficiency and aging as the experimental animals were 13 months old, post 10 months of osteoporosis induction.

Osteoporosis is reported to mainly affect the areas where bone remodelling is higher ${ }^{21}$, such as the proximal tibial metaphysis. Histological sections of proximal tibiae of OLI rats exhibited a prominent disruption in the trabecular bone, indicating that ovariectomy was successful. Qualitative and quantitative parameters measured using micro CT further substantiated the bone loss. Pathology of osteoporosis in humans is characterised by a reduction in the trabecular number and an increase in the trabecular separation ${ }^{22}$. Most of the animal models reviewed, exhibited similar trends in trabecular microarchitecture and demonstrated trabecular bone loss ${ }^{23}$. Both OSI and OLI rats exhibited a significant bone loss compared to Control animals, but it was well evident that the extent of bone loss increased with an increase of induction period in the OLI rats. Bone density was significantly reduced in the OLI rats supporting the hypothesis of long term osteoporosis induction and bone loss.

Meyer et al. reported that prolonged osteoporosis significantly impairs the process of fracture healing ${ }^{24}$. In ovariectomised rats, alteration in the expression of cytokines and growth factors occurs, which eventually alters the mineral matrix composition of the bone leading to impaired bone healing 25 . Osteoinductive capacity of bone matrix also decreases with age ${ }^{27}$. Plastic embedded bone sections stained with Stevenal's blue and van Gieson's picrofuchsin depicted impaired healing ability in the OLI model as the de novo bone formed within the defect area exhibited a loose arrangement of the osteoid matrix, resembling islands of woven bone ${ }^{28}$ with large void space in between and scarce cellular infiltration. Oliver et al., 2013 has also reported the significance of using a long term estrogendeficient rat model for understanding the pathophysiology of osteoporotic fracture healing in an open fracture model ${ }^{26}$.

Impaired proliferation and differentiation potential of resident mesenchymal stem cells and induction factors have been reported to delay bone healing ${ }^{29}$. In short term-induced models, delay in fracture healing is attributed to the accelerated resorption by osteoclast cells resulting in bone loss, but these models lack the reduction in osteogenic capacity of the bone-forming cells ${ }^{30}$. Conversely, in our study impaired osteogenic potential was evident in the long term induced osteoporotic model by 8 weeks, whereas the Control rats exhibited a complete bridging of the defect. Conclusive statements regarding the effect of aging on the OLI model can be made by conducting a more extensive study including extended time points and evaluating exclusive age related parameters. More information on the effect of long term induction on the mechanical parameters and molecular pathways may help in better validating the model for preclinical evaluations and further research is warranted.

\section{CONCLUSION}

The results of the study show that a long term osteoporosis induction period following ovariectomy in rats, induces chronic bone loss. This is attributed to the combined effect of ovariectomy and aging, thus mimicking the clinical pathology that exists among the geriatric population. The OLI models also exhibited a delayed healing pathology, further validating the osteoporotic model. Such OLI models may be used in future studies to assess the safety and efficacy of drugs indented for the prevention and treatment of osteoporosis. OLI rat models with inherent delayed healing ability may also be suitable for evaluating the in vivo osteogenic efficacy of therapeutic implants for addressing osteoporotic skeletal reconstructive surgeries.

\section{ACKNOWLEDGMENTS}

The authors acknowledge The Director, SCTIMST and The Head, BMT Wing, SCTIMST for the facilities provided. We acknowledge the support from Dr. Kalliyana Krishnan and Mr. Satheesh for the micro CT analysis and Mrs. Ganga, TEM lab for H\&E staining. Research grant to Dr. Annie John - "Catalyzed \& Supported by Science for Equity Empowerment and Development (SEED) Division, Department of Science \& Technology, New Delhi" and individual fellowship support to Mrs. Sunitha Chandran from Council of Scientific \& Industrial Research (CSIR), Government of India are thankfully acknowledged.

\section{Declaration of Conflicting Interests}

The author(s) declare no potential conflicts of interest with respect to the research, authorship, and/or publication of this article. 


\section{REFERENCES:}

1. Kanis JA, Borgstrom F, De Laet C, Johansson H, Johnell $\mathrm{O}$, Jonsson B, et al. Assessment of fracture risk. Osteoporos Int J Establ Result Coop Eur Found Osteoporos Natl Osteoporos Found USA. 2005; 16(6):581-9.

2. Colón-Emeric CS, Saag KG. Osteoporotic fractures in older adults. Best Pract Res Clin Rheumatol. 2006 Aug; 20(4):695-706.

3. Das S, Crockett JC. Osteoporosis - a current view of pharmacological prevention and treatment. Drug Des Devel Ther. 2013; 7:435-48.

4. Omi N, Ezawa I. [Animal models for bone and joint disease. Low calcium diet-induced rat model of osteoporosis]. Clin Calcium. 2011 Feb; 21(2):173-80.

5. Lasota A, Danowska-Klonowska D. Experimental osteoporosis--different methods of ovariectomy in female white rats. Rocz Akad Med W Białymst 1995. 2004; 49 Suppl 1:129-31.

6. Marie PJ, Kassem M. Extrinsic mechanisms involved in age-related defective bone formation. J Clin Endocrinol Metab. 2011 Mar; 96(3):600-9.

7. Jiang G, Matsumoto H, Fujii A. Mandible bone loss in osteoporosis rats. J Bone Miner Metab. 2003; 21(6):38895 .

8. Kubo T, Shiga T, Hashimoto J, Yoshioka M, Honjo H, Urabe M, et al. Osteoporosis influences the late period of fracture healing in a rat model prepared by ovariectomy and low calcium diet. J Steroid Biochem Mol Biol. 1999 Mar;68(5-6):197-202.

9. Rabbits $\mathrm{F}$ working group on revision of guidelines for health monitoring of rodents and, (Convenor) MM, Berard M, Feinstein R, Gallagher A, Illgen-Wilcke B, et al. FELASA recommendations for the health monitoring of mouse, rat, hamster, guinea pig and rabbit colonies in breeding and experimental units. Lab Anim. 2014 Jul $1 ; 48(3): 178-92$.

10. Kilkenny C, Browne W, Cuthill IC, Emerson M, Altman DG, National Centre for the Replacement, Refinement and Reduction of Amimals in Research. Animal research: reporting in vivo experiments--the ARRIVE guidelines. J Cereb Blood Flow Metab Off J Int Soc Cereb Blood Flow Metab. 2011 Apr;31(4):991-3.

11. Mohan BG, Shenoy SJ, Babu SS, Varma HK, John A. Strontium calcium phosphate for the repair of leporine (Oryctolagus cuniculus) ulna segmental defect. J Biomed Mater Res A. 2013 Jan; 101(1):261-71.

12. Habermann B, Kafchitsas K, Olender G, Augat P, Kurth A. Strontium Ranelate Enhances Callus Strength More Than PTH 1-34 in an Osteoporotic Rat Model of Fracture Healing. Calcif Tissue Int. 2010 Jan; 86(1):82-9.

13. Comelekoglu U, Bagis S, Yalin S, Ogenler O, Yildiz A, Sahin NO, et al. Biomechanical evaluation in osteoporosis: ovariectomized rat model. Clin Rheumatol. 2007 Mar; 26(3):380-4.

14. Kerschan-Schindl K, Mikosch P, Obermayer-Pietsch B, Gasser R, Dimai H-P, Fahrleitner-Pammer A, et al. Current Controversies in Clinical Management of Postmenopausal Osteoporosis. Exp Clin Endocrinol Diabetes. 2014 May 16; 122(08):437-44.

15. Syed FA, Melim T. Rodent models of aging bone: an update. Curr Osteoporos Rep. 2011 Dec; 9(4):219-28.
16. Dennison E, Cole Z, Cooper C. Diagnosis and epidemiology of osteoporosis. Curr Opin Rheumatol. 2005 Jul;17(4):456-61.

17. Kiebzak GM, Smith R, Howe JC, Gundberg CM, Sacktor B. Bone status of senescent female rats: chemical, morphometric, and biomechanical analyses. J Bone Miner Res Off J Am Soc Bone Miner Res. 1988 Aug; 3(4):43946.

18. Fukuda S, Iida H. Age-related changes in bone mineral density, cross-sectional area and the strength of long bones in the hind limbs and first lumbar vertebra in female Wistar rats. J Vet Med Sci Jpn Soc Vet Sci. 2004 Jul; 66(7):75560.

19. Vasikaran SD. Utility of biochemical markers of bone turnover and bone mineral density in management of osteoporosis. Crit Rev Clin Lab Sci. 2008; 45(2):221-58.

20. Sokoll LJ, Dawson-Hughes B. Effect of menopause and aging on serum total and ionized calcium and protein concentrations. Calcif Tissue Int. 1989 Mar; 44(3):181-5.

21. Compston J. Age-Related Changes in Bone Remodelling and Structure in Men: Histomorphometric Studies. J Osteoporos. 2011; 2011:1-4.

22. Laib DA, Barou O, Vico L, Lafage-Proust MH, Alexandre C, Rügsegger P. 3D micro-computed tomography of trabecular and cortical bone architecture with application to a rat model of immobilisation osteoporosis. Med Biol Eng Comput. 2000 May; 38(3):326-32.

23. Majumdar S, Genant HK, Grampp S, Newitt DC, Truong $\mathrm{V}-\mathrm{H}$, Lin JC, et al. Correlation of Trabecular Bone Structure with Age, Bone Mineral Density, and Osteoporotic Status: In Vivo Studies in the Distal Radius Using High Resolution Magnetic Resonance Imaging. J Bone Miner Res. 1997 Jan 1; 12(1):111-8.

24. Meyer RA, Tsahakis PJ, Martin DF, Banks DM, Harrow ME, Kiebzak GM. Age and ovariectomy impair both the normalization of mechanical properties and the accretion of mineral by the fracture callus in rats. J Orthop Res Off Publ Orthop Res Soc. 2001 May; 19(3):428-35.

25. Giannoudis P, Tzioupis C, Almalki T, Buckley R. Fracture healing in osteoporotic fractures: is it really different? A basic science perspective. Injury. 2007 Mar; 38 Suppl 1:S90-99.

26. Oliver RA, Yu Y, Yee G, Low AK, Diwan AD, Walsh WR. Poor histological healing of a femoral fracture following 12 months of oestrogen deficiency in rats. Osteoporos Int J Establ Result Coop Eur Found Osteoporos Natl Osteoporos Found USA. 2013 Oct; 24(10):2581-9.

27. Syftestad GT, Urist MR. Bone aging. Clin Orthop. 1982 Feb ; (162):288-97.

28. Shapiro F. Bone development and its relation to fracture repair. The role of mesenchymal osteoblasts and surface osteoblasts. Eur Cell Mater. 2008; 15:53-76.

29. Namkung-Matthai H, Appleyard R, Jansen J, Hao Lin J, Maastricht S, Swain M, et al. Osteoporosis influences the early period of fracture healing in a rat osteoporotic model. Bone. 2001 Jan; 28(1):80-6.

30. Chandran, S., Suresh B., Hari Krishanan VS, Varma, H.K. John A.. Osteogenic efficacy of strontium hydroxyapatite micro-granules in osteoporotic rat model. J. Biomater. Appl. 885328216647197. doi: $10.1177 / 0885328216647197$ 